\title{
Overexpression of HHLA2, a member of the B7 family, is associated with worse survival in human colorectal carcinoma
}

This article was published in the following Dove Press journal:

OncoTargets and Therapy

\author{
Ziwen Zhu'-3 \\ Weiguo Dong ${ }^{1-3}$ \\ 'Department of Gastroenterology, \\ Renmin Hospital of Wuhan University, \\ Wuhan, People's Republic of \\ China; ${ }^{2}$ Hubei Key Laboratory of \\ Digestive System Disease, Wuhan, \\ People's Republic of China; ${ }^{3}$ Central \\ Laboratory, Renmin Hospital of \\ Wuhan University, Wuhan, People's \\ Republic of China
}

Background: Colorectal carcinoma (CRC) is one of the most common malignancies, and immunotherapy has opened a new field of cancer treatment in recent years. Generally, CRC does not benefit from immunotherapy. HHLA2, a member of the B7 family, is a novel immune checkpoint molecule, and the prognostic value of HHLA2 in CRC patients and the association between HHLA2 expression and clinicopathological characteristics remains unknown.

Materials and methods: This study included 63 patients diagnosed with $\mathrm{CRC}$, and their resected specimens were obtained and constructed as a tissue microarray. Expression of HHLA2 and CD8 was detected by the double immunohistochemistry method. Based on follow-up data, correlations of HHLA2 expression and clinicopathological features, including overall survival, in CRC patients were evaluated.

Results: High HHLA2 expression was detected in CRC tumor tissues, compared to the adjacent noncancerous tissues. HHLA2 expression level was significantly related to the depth of invasion $(P=0.044)$ and $\mathrm{CD} 8^{+} \mathrm{T}$-cell infiltration status $(P=0.016)$, and predicted high mortality rate $(P=0.035)$. HHLA2 acted as an independent predictive factor in the overall survival of CRC patients $(P=0.039$, hazard ratio $=2.162,95 \%$ CI 1.041-3.084).

Conclusion: HHLA2 expression is upregulated in CRC patients, and HHLA2 is an independent prognostic factor of overall survival of CRC patients. High HHLA2 expression is closely correlated with CD8 T-cell infiltration status and can predict poor prognosis in CRC patients.

Keywords: colorectal carcinoma, HHLA2, immune checkpoint, prognosis

\section{Introduction}

Colorectal carcinoma (CRC) is one of the most common malignancies in the US, as well as the second cause of cancer death in men and the third in women. ${ }^{1}$ Despite a decline in the incidence, the distant 5-year relative survival rate for CRC is $14 \% .{ }^{2}$ It is necessary to find novel strategies to improve the survival of patients with CRC. Besides the therapeutic options of chemotherapy, radiotherapy, surgery, and targeted therapy, immunotherapy has opened a new field of cancer treatment in recent years. During tumorigenesis and cancer progression, immune cells decrease tumor immunogenicity in a process known as immunoediting, while tumors establish a microenvironment that actively suppresses an immune response. ${ }^{3}$ Thus, the interaction between the tumor microenvironment and immune system has exploited a new direction in cancer research. $^{4}$

Immune checkpoints are critical molecules that either up- or downregulate T-cell function. ${ }^{5}$ Immune checkpoint therapy - which targets regulatory pathways in T cells to enhance antitumor immune responses - has provided novel treatments against cancer. ${ }^{6}$
Correspondence: Weiguo Dong Department of Gastroenterology, Renmin Hospital of Wuhan University, Wuhan, Hubei Province, People's Republic of China Tel +86 278804 I9II

Fax +862788042292

Email dongweiguo@whu.edu.cn $\mathrm{BY}$
NC hereby accept the Terms. Non-commercial uses of the work are permitted without any further permission from Dove Medict
for commercial use of this work, please see paragraphs 4.2 and 5 of our Terms (https://www.dovepress.com/terms.php). 
Recent research has found that ligands and receptors of the B7/CD28 family have immunoregulatory function in cancer pathogenesis. The B7 family now comprises at least 10 members, including B7-1, B7-2, CD28, ICOS-L, programmed death ligand 1 (PD-L1) and 2 (PD-L2), B7-H3, $\mathrm{B} 7 \mathrm{x}, \mathrm{B} 7-\mathrm{H} 6$, and human endogenous retrovirus-H long terminal repeat-associating protein 2 (HHLA2). ${ }^{7}$ Among these ligands, PD-L1 and PD-L2 represent two ligands for the programmed death 1 (PD-1) receptor. ${ }^{8}$ These molecules function as antigen-presenting cells (APCs), and modulate T-cell proliferation and function. However, the overexpression of PD-L1 and PD-1 on tumor cells and tumor-infiltrating lymphocytes (TILs) can inhibit T-cell activation and help tumor cells evade immune elimination, and are related to poor outcome in some human cancers. ${ }^{9-14}$ Inhibition of the PD-1/ PD-L1 pathway by monoclonal antibodies has been used in some clinical trials, and the Food and Drug Administration (FDA) has approved these immune checkpoint agents for treating melanoma, non-small cell lung cancer (NSCLC), and other cancers. ${ }^{15}$

HHLA2 is a member of the B7 family and suppresses CD4 and CD8 T-cell function in the presence of T-cell receptor signaling. ${ }^{16-18}$ In addition, HHLA2 significantly reduces cytokine production by $\mathrm{T}$ cells including interferon gamma (IFN $\gamma$ ), TNF- $\alpha$, interleukin (IL)-5, IL-10, IL-13, IL-17A, and IL-22. ${ }^{19}$ Recent research has reported that HHLA2 has limited expression in normal tissues, but is widely expressed in different human cancers. ${ }^{20}$ However, the prognostic value of HHLA2 in patients with CRC and the association between HHLA2 and clinicopathological characteristics remains unknown.

Generally, CRC has not benefited from immunotherapy. ${ }^{21}$ In this research, $\mathrm{CRC}$ specimens were obtained from surgical patients and were constructed as a tissue microarray (TMA). HHLA2 and CD8 expressions were detected by immunohistochemistry (IHC) and double IHC. On the basis of follow-up data, the correlations of HHLA2 expression and clinicopathological features, including overall survival in patients with CRC, were evaluated.

\section{Materials and methods}

\section{Ethics statement}

Ethical approval was given by the Medical Ethics Committee of Renmin Hospital, Wuhan University. Patients provided written informed consent for study participation and the use of their tissues for future research prior to treatment. All procedures conducted in studies involving human participants were in accordance with the ethical standards of the institutional and/or national research committee and with the 1964 Helsinki declaration and its later amendments or comparable ethical standards.

\section{Patients and follow-up}

In total, 75 patients diagnosed with $\mathrm{CRC}$ in the period from September 2005 to July 2013 and treated surgically in the Renmin Hospital of Wuhan University were included in this study. Twelve patients who received chemotherapy, radiotherapy, or neoadjuvant therapy before surgery were excluded from the study. Thus, 63 patients with CRC were included in this study. Formalin-fixed, paraffin-embedded (FFPE) tissues were collected from the Department of Pathology, Renmin Hospital of Wuhan University.

The follow-up began on the date of surgery and ended in August 2016. Clinicopathological parameters, including age, gender, survival status, smoking history, alcohol consumption history, as well as T, N, M, and American Joint Committee on Cancer (AJCC) stage, were recorded. CRC specimens had been classified according to the 7 th edition of TNM classification specified by the Union for International Cancer Control/American Joint Committee on Cancer (2010). Overall survival was defined as the period from initial diagnosis to death or the end of follow-up. Thirty-two patients $(50.8 \%)$ died during the follow-up period, and 31 patients (49.2\%) were alive on the date of last follow-up. Mean overall survival was 61 months and ranged from 1 to 132 months.

\section{TMA construction and IHC analysis}

FFPE CRC tissues and corresponding adjacent noncancerous tissues were collected, and hematoxylin- and eosin-stained slides were screened by two pathologists independently to confirm the diagnosis of CRC. The most representative tumor tissues and noncancerous tissues were selected to construct the TMA slide. As described in a previous study, ${ }^{22}$ a $1.5-\mathrm{mm}$ diameter core for each sample was punched into the TMA. Finally, the TMA comprised $63 \mathrm{CRC}$ tissues and matched noncancerous tissue for each.

TMA sections were deparaffinized, and was followed by antigen retrieval treatment with EDTA ( $1 \mathrm{mM}, \mathrm{pH} 8.0)$ in a microwave oven for $20 \mathrm{~min}$. The rabbit anti-human HHLA2 polyclonal antibody (1:200 dilution, ab214327, Abcam, Cambridge, UK) and mouse anti-human CD8 monoclonal antibody (mAb; C8/144B; ready-to-use, Dako, Carpinteria, CA, USA) were used for overnight incubation at $4^{\circ} \mathrm{C}$. Then, a Dako EnVision system - a horseradish peroxidase (HRP)-conjugated polymer backbone, which also carries secondary antibody molecules directed against rabbit and 
mouse immunoglobulin $\mathrm{G}$ (IgG) - was applied, followed by 3, 3'-diaminobenzidine (DAB) chromogen (Dako Corporation) and hematoxylin nuclear counterstaining. The HHLA2 polyclonal antibody is a synthetic peptide within Human HHLA2 aa 270-315 conjugated to keyhole limpet hemocyanin.

Double IHC was conducted to detect the HHLA2 and CD8 co-expressions. The FFPE TMAs were deparaffinized and rehydrated, followed by antigen retrieval with EDTA ( $1 \mathrm{mM}$, $\mathrm{pH} \mathrm{8.0)} \mathrm{in} \mathrm{a} \mathrm{microwave} \mathrm{oven} \mathrm{for} 20 \mathrm{~min}$. The rabbit anti-human HHLA2 polyclonal antibody (1:200 dilution, ab214327, Abcam, USA) and mouse anti-human CD8 mAb (C8/144B; ready-to-use, Dako, Carpinteria, $\mathrm{CA}$ ) were used for overnight incubation at $4{ }^{\circ} \mathrm{C}$. The goat anti-rabbit secondary antibody conjugated with HRP and goat anti-mouse secondary antibody conjugated with alkaline phosphatase (AP) were added. Thereafter, the TMA was incubated with DAB and permanent red as the chromogenic substrate, respectively. Finally, hematoxylin was applied as a nuclear counterstain.

The signals of labeling cells were observed under microscopy (Olympus BX52, CCD DP80) and evaluated by two independent researchers who were blinded to the clinicopathological factors of the study. The expression of HHLA2 was recorded with $\mathrm{H}$-score in a semi-quantitation method according to the area of positivity (AP) and the intensity of staining (IS). AP depended on the percentage of positivestained cells as follows: $0(0 \%), 1(1 \%-25 \%), 2(26 \%-50 \%)$, $3(51 \%-75 \%)$, and $4(>75 \%)$. IS was graded as 0 (negative), 1 (weak), 2 (moderate), and 3 (strong). The final H-score was calculated by the equation: $\mathrm{H}$-score $=\mathrm{AP} \times \mathrm{IS}$. Additionally, the number of positive cells was counted in each core of TMA for the CD8 evaluation. The cutoff point of high or low expression was determined on the median of total scores.

\section{Statistical analysis}

All statistical analyses were carried out by SPSS v19.0 (Chicago, IL, USA). The correlations of HHLA2 expression and clinicopathological parameters were assessed using the $\chi^{2}$ test or Fisher exact test as appropriate. The survival analysis was examined by the Kaplan-Meier curve, and a log-rank test was conducted to determine the statistical difference survival data. Student's $t$-test was conducted to determine the association between CD8 T-cell counts and HHLA2 expression. Univariate and multivariate Cox proportion hazard regression models of survival were created to evaluate the prognostic values of clinicopathological parameters and HHLA2 expression. Two-tailed $P<0.05$ was considered to be statistically significant.

\section{Results \\ Patient characteristics}

Among the 63 patients with CRC, 39 (61.9\%) were male and $24(38.1 \%$ ) were female, with mean age of 62 (range 29-89) years. At the end of follow-up, $32(50.8 \%)$ patients died and $31(49.2 \%)$ patients were alive. Eight (12.7\%) patients had a history of smoking and seven (11.1\%) had a history of alcohol consumption. According to the depth of tumor invasion, 25 (39.7\%) were T2, 37 (58.7\%) were T3, and 1 (1.6\%) was T4. For the lymph node metastasis, 37 (58.7\%) were N0, $19(30.2 \%)$ were N1, and $7(11.1 \%)$ were N2. In the aspect of distant metastasis, $60(95.2 \%)$ were M0, and $3(4.8 \%)$ were M1. Further, 23 (36.5\%) patients were classified as Stage I, $27(42.9 \%)$ as Stage II, and $13(20.6 \%)$ as Stage III according to the 8 th AJCC staging system (Table 1).

\section{HHLA2 expression in CRC and noncancerous tissues}

HHLA2 expression at the protein level in CRC tissues is almost unknown at present. As shown in Figure 1, HHLA2 protein was expressed in both CRC and noncancerous tissues. In the adjacent noncancerous tissues, HHLA2 was found to be expressed in the intestinal epithelium and macrophages (Figure 1A and B). In cancer tissues, HHLA2 was located on the membrane and cytoplasm of tumor cells, and was also found in the fibroblasts of the stroma (Figure 1C). However,

Table I Patient characteristics $(\mathrm{N}=63)$

\begin{tabular}{lll}
\hline Characteristic & Sub-characteristic & Value (\%) \\
\hline Age & Male & 62 (range 29-89) \\
Gender & Female & $39(61.9)$ \\
Survival status & Death & $24(38.1)$ \\
Depth of invasion (T) & Survival & $32(50.8)$ \\
& T2 & $31(49.2)$ \\
Lymph node metastasis (N) & T3 & $25(39.7)$ \\
& NI & $37(58.7)$ \\
Distant metastasis (M) & N2 & $1(1.6)$ \\
AJCC stage & M0 & $37(58.7)$ \\
& MI & $19(30.2)$ \\
Smoking history & I & $7(11.1)$ \\
& II & $60(95.2)$ \\
Alcohol history & III & $3(4.8)$ \\
& & $23(36.5)$ \\
Total & Yes & $27(42.9)$ \\
\hline
\end{tabular}

Abbreviation: AJCC, American Joint Committee on Cancer. 


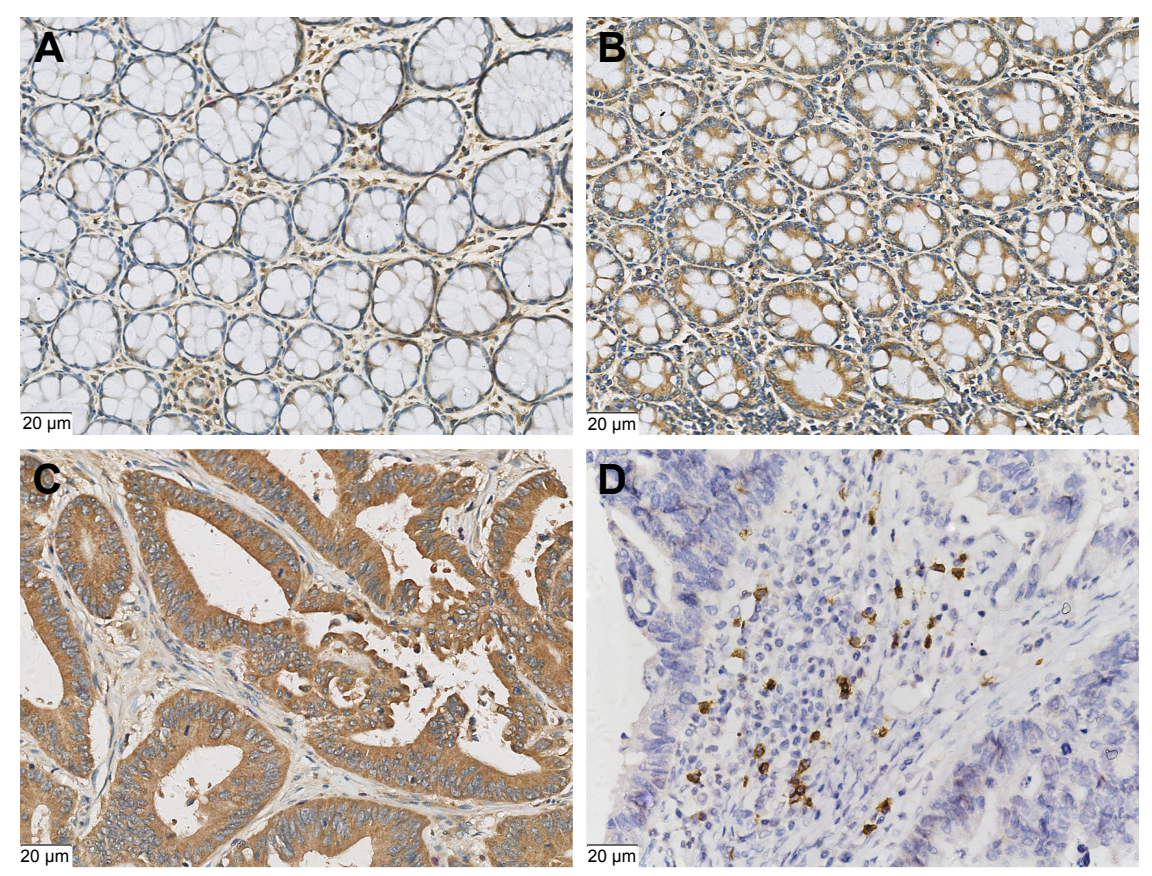

Figure I Representative photomicrographs of HHLA2 expression and CD8-positive T-cell infiltration in colorectal lesions.

Notes: (A) Weak expression of HHLA2 in the adjacent intestinal epithelium tissues. (B) Positive expression of HHLA2 in the adjacent intestinal epithelium tissues. (C) High expression of HHLA2 in the CRC tissues. (D) CD8-positive expression in the T cells of stroma. (Original magnification: 400x).

the staining intensity of HHLA2 in noncancerous tissues was obviously weaker than that in CRC tissues. CD8 was positive at the cellular membrane of cytotoxic $\mathrm{T}$ lymphocytes (Figure 1D).

The H-score of HHLA2 ranged from 2.0 to 12.0 and a median of 9.0. The median score was used to determine the cutoff value of high or low HHLA2 expression level. An $\mathrm{H}$-score $>9.0$ was defined as high HHLA2 expression, and $\mathrm{H}$-score $\leq 9.0$ indicated low HHLA2 expression. In 63 CRC tissues, 47.6\% (30/63) showed high HHLA2 expression, and $52.4 \%(33 / 63)$ cases of low HHLA2 expression was found.

The mean value of CD8 T-cell numbers was 362.3 and the median value was 244.0 (range 100-1,830). A CD8 T-cell count $>244.0$ was regarded as positive, and counts $\leq 244.0$ were negative. Among 63 patients with CRC, 32 (50.8\%) cases were CD8 negative and $31(49.2 \%)$ cases were CD8 positive.

\section{Expression of HHLA2 in CRC patients and clinicopathological variables}

The association between HHLA2 expression and clinicopathological parameters in CRC was investigated by $\chi^{2}$ test. As listed in Table 2, significant correlation was found between the depth of invasion (T) and HHLA2 expression $(P=0.044)$, whereas HHLA2 expression was not significantly
Table 2 Correlations between HHLA2 expression and clinicopathological parameters of patients with CRC

\begin{tabular}{|c|c|c|c|c|}
\hline \multirow[t]{2}{*}{ Characteristic } & \multirow[t]{2}{*}{$\mathbf{N}$} & \multicolumn{3}{|l|}{ HHLA2 } \\
\hline & & Low (\%) & High (\%) & $P$-value \\
\hline \multicolumn{4}{|l|}{ Gender } & 0.414 \\
\hline Male & 39 & $22(34.9)$ & $17(27.0)$ & \\
\hline Female & 21 & II (I7.5) & $13(20.6)$ & \\
\hline \multicolumn{4}{|l|}{ Age } & 0.374 \\
\hline$\leq 64$ years & 32 & $15(23.8)$ & $17(27.0)$ & \\
\hline$>64$ years & 31 & $18(28.6)$ & $13(20.6)$ & \\
\hline \multicolumn{4}{|c|}{ Depth of invasion (T) } & 0.044 \\
\hline $\mathrm{T} 2$ & 25 & I 7 (27.0) & $8(12.7)$ & \\
\hline T3, T4 & 38 & $16(25.4)$ & $22(34.9)$ & \\
\hline \multicolumn{4}{|c|}{ Lymph node metastasis $(\mathrm{N})$} & 0.845 \\
\hline No & 37 & $19(30.2)$ & $18(28.6)$ & \\
\hline NI, N2 & 26 & $14(22.2)$ & $12(19.0)$ & \\
\hline \multicolumn{4}{|c|}{ Distant metastasis $(\mathrm{M})$} & 0.063 \\
\hline MO & 60 & $33(52.4)$ & $27(42.9)$ & \\
\hline MI & 3 & $0(0.0)$ & $3(4.8)$ & \\
\hline \multicolumn{4}{|l|}{ AJCC stage } & 0.064 \\
\hline I, II & 50 & $23(36.5)$ & 27 (42.9) & \\
\hline III & 13 & $10(15.9)$ & $3(4.8)$ & \\
\hline \multicolumn{4}{|l|}{ Smoking history } & 0.710 \\
\hline Yes & 8 & $5(7.9)$ & $3(4.8)$ & \\
\hline No & 55 & $28(44.4)$ & 27 (42.9) & \\
\hline \multicolumn{4}{|l|}{ Alcohol history } & 1.000 \\
\hline Yes & 7 & $4(6.3)$ & $3(4.8)$ & \\
\hline No & 56 & $29(46.0)$ & $27(42.9)$ & \\
\hline \multicolumn{4}{|l|}{ CD8 status } & 0.016 \\
\hline Negative & 32 & $12(19.0)$ & 20 (3।.7) & \\
\hline Positive & 31 & $21(33.3)$ & $10(15.9)$ & \\
\hline \multicolumn{2}{|l|}{ Total } & $33(52.4)$ & $30(47.6)$ & $63(100)$ \\
\hline
\end{tabular}

Note: Bold figures represent statistically significant $P<0.05$.

Abbreviation: $\mathrm{CRC}$, colorectal carcinoma. 

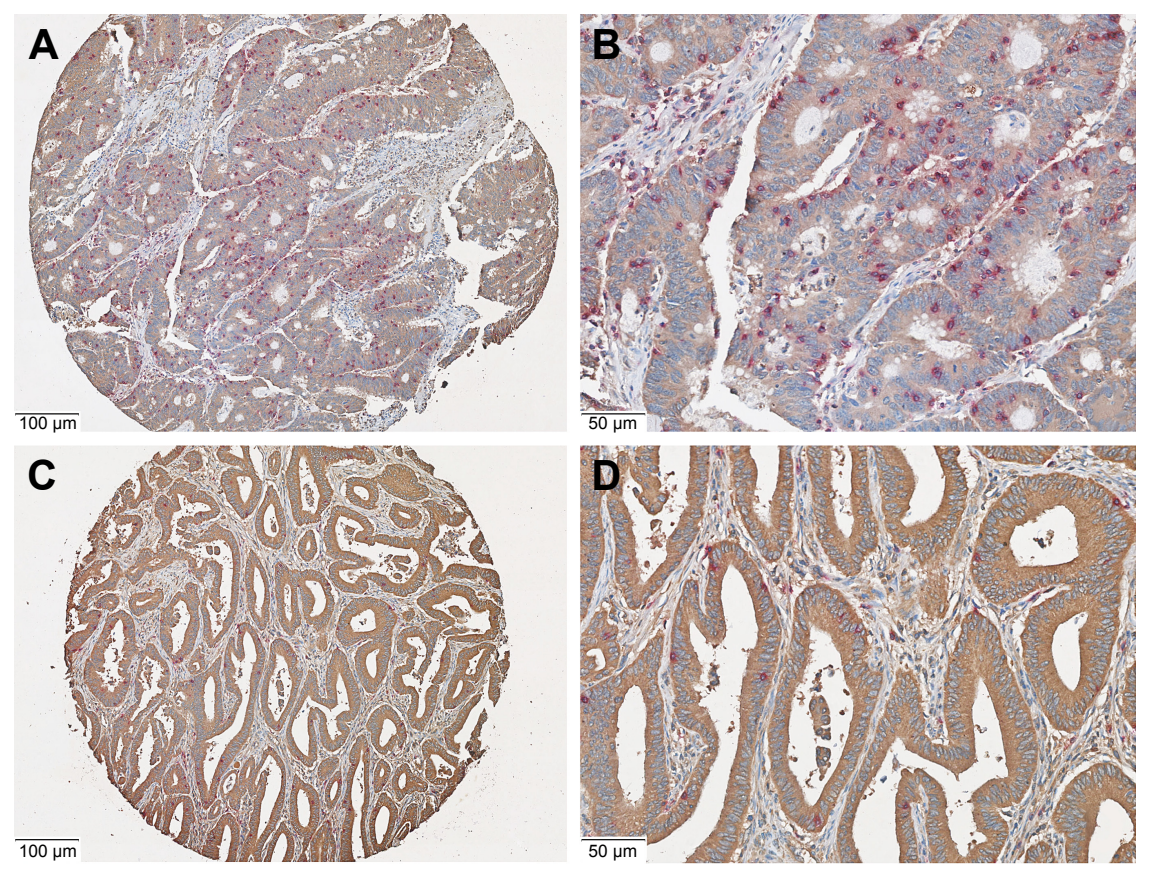

Figure 2 Co-expression of HHLA2 and CD8 in the CRC tissues.

Notes: (A and B) Low expression of HHLA2 (brown signal) and high CD8 (purple signal) in the same CRC tissues. (C and D) High expression of HHLA2 and low CD8 in the same CRC tissues. (Original magnification: A, C I00×; B, D 200×).

Abbreviation: CRC, colorectal carcinoma.

associated with age, gender, lymph node metastasis $(\mathrm{N})$, distant metastasis (M), stage, and smoking or alcohol history in patients with CRC.

\section{Relationship between HHLA2 expression and CD8 T-cell infiltration}

The results of $\chi^{2}$ test illustrated that HHLA2 expression was correlated with CD8 T-cell infiltration in patients with CRC ( $P=0.016$, Table 2). Further, the relationship between CD8 T-cell infiltration status in patients with CRC and HHLA2 expression was examined by two independent $t$-tests. The number of CD8-positive cells in the HHLA2 high-expression group was significantly lower than the number in the HHLA2 low-expression group $(P=0.023$, Figures $2 \mathrm{~A}-\mathrm{D}$ and 3$)$.

\section{Prognostic value of HHLA2 expressions in $C R C$ patients}

Subsequently, the prognostic value of HHLA2 expression in CRC was investigated. Survival analysis determined by the Kaplan-Meier curve and log-rank test demonstrated that high expression of HHLA2 predicted poorer survival and high mortality rate in patients with CRC (Figure 4A, $P=0.035$ ). However, high expression of HHLA2 and low CD8 were found to have no significant prognostic value for the overall survival of patients with $\mathrm{CRC}(P=0.185$, Figure 4B).
Univariate and multivariate analyses of the Cox proportional hazard model on overall survival was carried out to determine the prognostic value of HHLA2 expression and other clinicopathological variables. In the univariate analysis, HHLA2 expression showed significant correlation to the survival of patients with CRC ( $\mathrm{HR}=2.249,95 \%$ $\mathrm{CI}=10.97-4.610, P=0.027)$. Meanwhile, depth of invasion (HR=2.231, 95\% CI=1.017-4.897, $P=0.045)$, lymph node metastasis $(\mathrm{HR}=2.945,95 \% \mathrm{CI}=1.447-5.996, P=0.003)$, distant metastasis $(\mathrm{HR}=4.753,95 \% \mathrm{CI}=1.093-20.675, P=0.038)$,

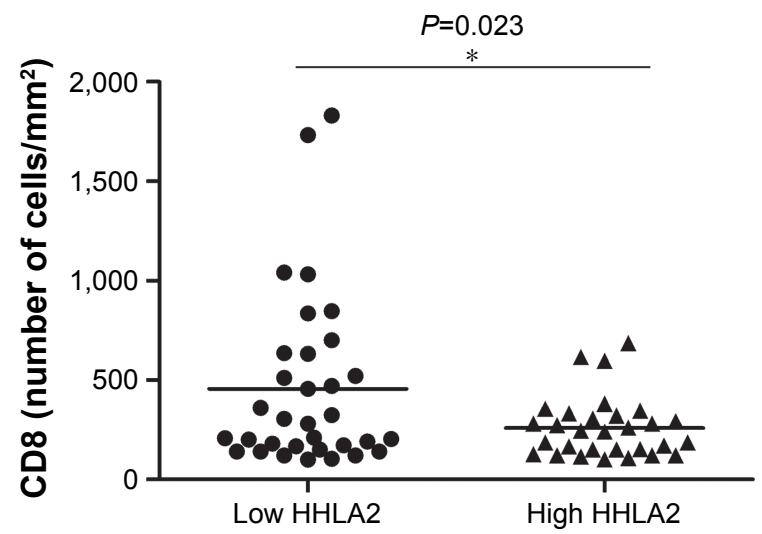

Figure 3 CD8-positive cell-infiltrating status in CRC patients with HHLA2 high or low expression.

Notes: The number of CD8-positive cells in the high-HHLA2 group was significantly lower than the number in the low-HHLA2 group. ${ }^{*} P=0.023$ by two independent $t$-tests. Abbreviation: CRC, colorectal carcinoma. 

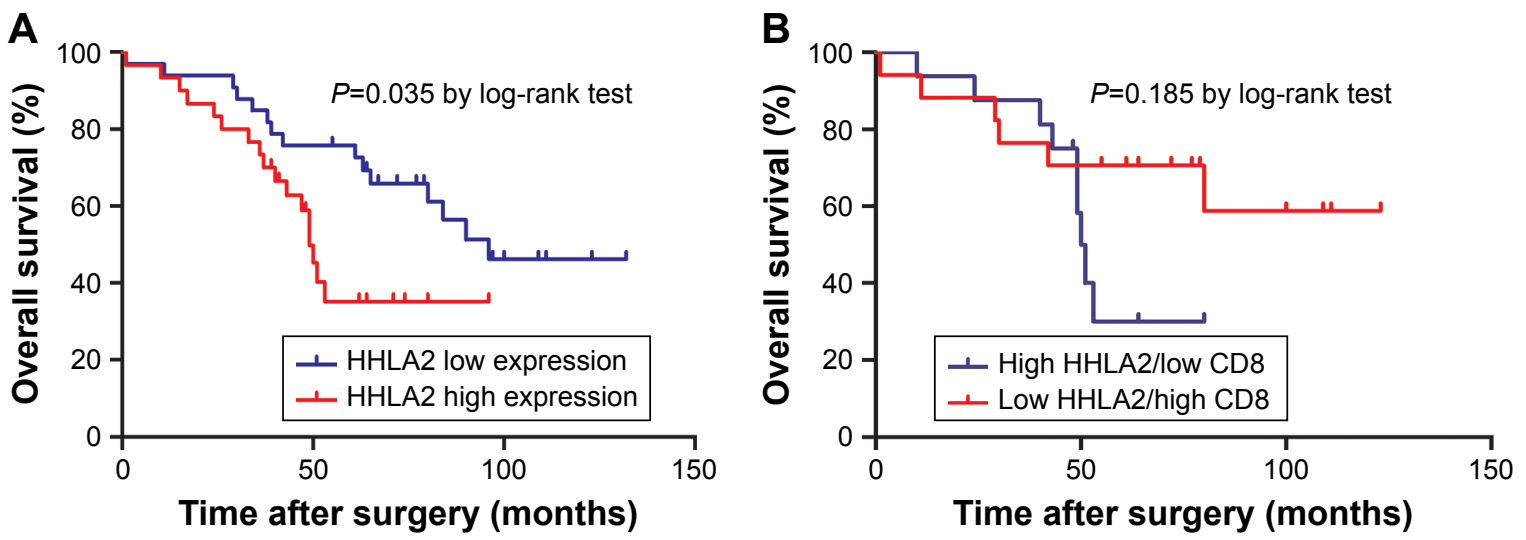

Figure 4 Correlation of HHLA2 expression level and overall survival in patients with CRC.

Notes: (A) Thirty-three patients with low HHLA2 expression showed better overall survival than 30 patients with high HHLA2 expression ( $P=0.035$ ). (B) High expression of HHLA2 and low CD8 showed no significant prognostic value for the overall survival of patients with $C R C(P=0.185)$.

Abbreviation: CRC, colorectal carcinoma.

and AJCC stage $(\mathrm{HR}=2.169,95 \% \mathrm{CI}=1.198-3.928, P=0.011)$ were found to be associated with survival (Table 3 ).

The independent prognostic value was detected by multivariate analysis (Table 3 ). The results indicated that HHLA2 expression $(\mathrm{HR}=2.162,95 \% \mathrm{CI}=1.041-3.084, P=0.039)$ and lymph node metastasis $(\mathrm{HR}=2.992,95 \% \mathrm{CI}=1.426-6.279$, $P=0.004$ ) were two independent prognosis factors in the overall survival of patients with CRC. However, depth of invasion, distant metastasis, AJCC stage, and CD8-positive cell-infiltration status were not independent of the overall survival in our research.

\section{Discussion}

This is the first study reporting the clinical significance of HHLA2 in CRC, and demonstrates that HHLA2 might be a novel immunosuppressive mechanism within the colon cancer microenvironment as well as a new target for CRC immunotherapy. The HHLA2 pathway represents a novel immunosuppressive mechanism within the tumor microenvironment and an attractive target for human cancer therapy. Here, we reported that HHLA2 was overexpressed in the tumor tissues, compared to the adjacent noncancerous tissues, in patients with CRC. The HHLA2 expression level

Table 3 COX proportional hazard models on overall survival of patients

\begin{tabular}{|c|c|c|c|c|}
\hline \multicolumn{5}{|c|}{ COX proportional hazard models } \\
\hline \multirow[t]{2}{*}{ Factors } & \multicolumn{2}{|c|}{ Univariate analysis } & \multicolumn{2}{|c|}{ Multivariate analysis } \\
\hline & $P$-value & HR (95\% Cl) & $P$-value & HR (95\% Cl) \\
\hline \multicolumn{5}{|l|}{ Gender } \\
\hline Male vs female & 0.808 & $0.915(0.446-1.876)$ & & \\
\hline \multicolumn{5}{|l|}{ Age } \\
\hline$\leq 64$ vs $>64$ & 0.253 & $1.510(0.745-4.492)$ & & \\
\hline \multicolumn{5}{|c|}{ HHLA2 expression } \\
\hline Low vs high & 0.027 & $2.249(10.97-4.610)$ & 0.039 & $2.162(1.04 I-3.084)$ \\
\hline \multicolumn{5}{|l|}{ CD8 status } \\
\hline Low vs high & $0.5 \mathrm{II}$ & $0.789(0.390-1.598)$ & & \\
\hline \multicolumn{5}{|c|}{ Depth of invasion $(T)$} \\
\hline $\mathrm{T} 2$ vs T3, T4 & 0.045 & $2.231(1.017-4.897)$ & 0.242 & I.643 (0.7I5-3.77I) \\
\hline \multicolumn{5}{|c|}{ Lymph node metastasis $(\mathrm{N})$} \\
\hline N0 vs NI, N2 & 0.003 & $2.945(1.447-5.996)$ & 0.004 & $2.992(1.426-6.279)$ \\
\hline \multicolumn{5}{|c|}{ Distant metastasis (M) } \\
\hline MO vs MI & 0.038 & $4.753(1.093-20.675)$ & 0.073 & $4.195(0.876-20.086)$ \\
\hline \multicolumn{5}{|l|}{ AJCC stage } \\
\hline I, II vs III & 0.011 & $2.169(1.198-3.928)$ & 0.848 & $1.097(0.423-2.843)$ \\
\hline \multicolumn{5}{|l|}{ Smoking history } \\
\hline Yes vs no & 0.538 & $0.538(0.163-1.778)$ & & \\
\hline \multicolumn{5}{|l|}{ Alcohol history } \\
\hline Yes vs no & 0.098 & $0.185(0.025-1.363)$ & & \\
\hline
\end{tabular}


was significantly related to the depth of invasion and CD8 T-cell infiltration status. High expression of HHLA2 protein predicted a high mortality rate, and HHLA2 acted as an independent predictive factor in the overall survival of patients with CRC.

A total of 3,682,000 new cancer cases and 2,229,300 cancer deaths were estimated in the People's Republic of China in 2013. Lung cancer, liver cancer, stomach cancer, esophageal cancer, and CRC are the five leading causes of cancer death, accounting for $\sim 60 \%$ of all cancer deaths. ${ }^{23}$ Recently, major progress in characterization of immune landscape of tumor-associated microenvironment has been made, especially with respect to microsatellite status of CRC. ${ }^{24}$ Notwithstanding that HHLA2 is a novel immune checkpoint molecule that belongs to the $\mathrm{B} 7$ family of ligands, and its main function is to decrease both CD4 and CD8 T-cell proliferation and inhibit the production of T-cell cytokines, including IFN $\gamma$, TNF- $\alpha$, and ILs, ${ }^{19}$ its function in CRC is still unclear. In our research, the level of HHLA2 expression was related to the CD8 T-cell infiltration status, and the number of CD8-positive T cells in the high-HHLA2-expression group was significantly lower than the HHLA2-negative group. Although a previous study showed that HHLA2 played a co-stimulatory role and increased cytokine production, ${ }^{18}$ most studies have revealed that HHLA2 inhibits T-cell proliferation and function. ${ }^{19,25,26}$ Similarly, our results demonstrated that high expression of HHLA2 is associated with low CD8 TILs. This suggests that HHLA2 may predominantly functions as a T-cell co-inhibitory ligand.

Most normal tissues do not express HHLA2 except the placenta, gut, gallbladder, and breast whereas HHLA2 is widely expressed in a variety of cancer tissues. ${ }^{20,25}$ In this study, we confirmed that HHLA2 was expressed in both noncancerous intestinal specimens and cancer specimens from patients with CRC. However, the IHC staining intensity in tumor tissues was stronger than in noncancerous intestinal tissues, which illustrated that HHLA2 is upregulated in tumor cells of CRC. Moreover, we found that the high expression of HHLA2 was associated with poor prognostic features in patients with $\mathrm{CRC}$, which is consistent with previous studies of human lung cancer, breast cancer, and osteosarcoma. ${ }^{20,27,28}$

It was noted that the CD8-TILs status was not a predictive value in patient prognosis in this research. Actually, TILs are prognostic in some cancers, but the predictive value of TILs remains controversial in CRC and in other types of cancer. Although TIL density has been found to be independently prognostic and associated with lymph node harvest and lymph node size in $\mathrm{CRC},{ }^{29}$ a recent study investigated the PD-L1 expression in patients with Stage III CRC and demonstrated that the intratumoral CD8-positive T-cell count was related to prognosis, but was not an independent factor; however, peritumoral CD8-positive T cells had no statistically predictive value in disease-free survival. ${ }^{30}$ This controversial phenomenon has also been discovered in lung cancer. ${ }^{27,31,32}$ Taken together, there may be other factors implicated in TIL-infiltration status, and HHLA2 expression may partly be a response to the T-cell infiltration in CRC and other cancers.

In conclusion, we demonstrated that HHLA2 is upregulated in patients with CRC and first reported that HHLA2 is an independent prognostic factor of the overall survival of patients with CRC. High expression of HHLA2 is correlated with CD8 T-cell infiltrating status, and predicted a low survival rate in patients with CRC. Our results illustrated that HHLA2 is a critical immune checkpoint in CRC, and further studies are required to elucidate the mechanisms of HHLA2 overexpression and its therapeutic values.

Because HHLA2 can suppress T-cell function, the upregulation of HHLA2 expression on tumor cells and inducible HHLA2 expression on APCs provide a novel mechanism for tumor immune evasion. Therefore, HHLA2 could be an attractive target for human cancer immunotherapy.

\section{Disclosure}

The authors report no conflicts of interest in this work.

\section{References}

1. Siegel RL, Miller KD, Jemal A. Cancer statistics, 2017. CA Cancer J Clin. 2017;67(1):7-30.

2. Siegel RL, Miller KD, Fedewa SA, et al. Colorectal cancer statistics, 2017. CA Cancer J Clin. 2017;67(3):177-193.

3. Markman JL, Shiao SL. Impact of the immune system and immunotherapy in colorectal cancer. J Gastrointest Oncol. 2015;6(2):208-223.

4. Lynch D, Murphy A. The emerging role of immunotherapy in colorectal cancer. Ann Transl Med. 2016;4(16):305.

5. Sharpe AH, Abbas AK. T-cell costimulation - biology, therapeutic potential, and challenges. N Engl J Med. 2006;355(10):973-975.

6. Sharma P, Allison JP. The future of immune checkpoint therapy. Science. 2015;348(6230):56-61.

7. Ni L, Dong C. New checkpoints in cancer immunotherapy. Immunol Rev. 2017;276(1):52-65

8. Leung J, Suh WK. The CD28-B7 family in anti-tumor immunity: emerging concepts in cancer immunotherapy. Immune Netw. 2014;14(6): 265-276.

9. Parry RV, Chemnitz JM, Frauwirth KA, et al. CTLA-4 and PD-1 receptors inhibit T-cell activation by distinct mechanisms. Mol Cell Biol. 2005;25(21):9543-9553.

10. Keir ME, Butte MJ, Freeman GJ, Sharpe AH. PD-1 and its ligands in tolerance and immunity. Annu Rev Immunol. 2008;26:677-704.

11. Zang X, Allison JP. The B7 family and cancer therapy: costimulation and coinhibition. Clin Cancer Res. 2007;13(18 Pt 1):5271-5279. 
12. Ohaegbulam KC, Assal A, Lazar-Molnar E, Yao Y, Zang X. Human cancer immunotherapy with antibodies to the PD-1 and PD-L1 pathway. Trends Mol Med. 2015;21(1):24-33.

13. Ott PA, Hodi FS, Robert C. CTLA-4 and PD-1/PD-L1 blockade: new immunotherapeutic modalities with durable clinical benefit in melanoma patients. Clin Cancer Res. 2013;19(19):5300-5309.

14. Alsaab HO, Sau S, Alzhrani R, et al. PD-1 and PD-L1 checkpoint signaling inhibition for cancer immunotherapy: mechanism, combinations, and clinical outcome. Front Pharmacol. 2017;8:561.

15. Brahmer JR, Tykodi SS, Chow LQ, et al. Safety and activity of antiPD-L1 antibody in patients with advanced cancer. N Engl J Med. 2012; 366(26):2455-2465.

16. Mager DL, Hunter DG, Schertzer M, Freeman JD. Endogenous retroviruses provide the primary polyadenylation signal for two new human genes (HHLA2 and HHLA3). Genomics. 1999;59(3):255-263.

17. Flajnik MF, Tlapakova T, Criscitiello MF, Krylov V, Ohta Y. Evolution of the B7 family: co-evolution of B7H6 and NKp30, identification of a new B7 family member, B7H7, and of B7's historical relationship with the MHC. Immunogenetics. 2012;64(8):571-590.

18. Zhu Y, Yao S, Iliopoulou BP, et al. B7-H5 costimulates human T cells via CD28H. Nat Commun. 2013;4:2043.

19. Zhao RH, Chinai JM, Buhl S, et al. HHLA2 is a member of the B7 family and inhibits human CD4 and CD8 T-cell function. Proc Natl Acad Sci U S A. 2013;110(24):9879-9884.

20. Janakiram M, Chinai JM, Fineberg S, et al. Expression, clinical significance, and receptor identification of the newest B7 family member HHLA2 protein. Clin Cancer Res. 2015;21(10):2359-2366.

21. Boland PM, Ma WW. Immunotherapy for colorectal cancer. Cancers (Basel). 2017;9(5). pii: E50.

22. Kawakami F, Sircar K, Rodriguez-Canales J, et al. Programmed cell death ligand 1 and tumor-infiltrating lymphocyte status in patients with renal cell carcinoma and sarcomatoid dedifferentiation. Cancer. 2017;123(24):4823-4831
23. Chen W, Zheng R, Zhang S, et al. Cancer incidence and mortality in China, 2013. Cancer Lett. 2017;401:63-71.

24. Karpinski P, Rossowska J, Sasiadek MM. Immunological landscape of consensus clusters in colorectal cancer. Oncotarget. 2017;8(62): 105299-105311.

25. Janakiram M, Shah UA, Liu W, Zhao A, Schoenberg MP, Zang X. The third group of the B7-CD28 immune checkpoint family: HHLA2, TMIGD2, B7x, and B7-H3. Immunol Rev. 2017;276(1):26-39.

26. Schildberg FA, Klein SR, Freeman GJ, Sharpe AH. Coinhibitory pathways in the B7-CD28 ligand-receptor family. Immunity. 2016; 44(5):955-972.

27. Cheng H, Janakiram M, Borczuk A, et al. HHLA2, a new immune checkpoint member of the B7 family, is widely expressed in human lung cancer and associated with EGFR mutational status. Clin Cancer Res. 2017;23(3):825-832.

28. Koirala P, Roth ME, Gill J, et al. HHLA2, a member of the B7 family, is expressed in human osteosarcoma and is associated with metastases and worse survival. Sci Rep. 2016;6:31154.

29. Märkl B, Wieberneit J, Kretsinger H, et al. Number of intratumoral T lymphocytes is associated with lymph node size, lymph node harvest, and outcome in node-negative colon cancer. Am J Clin Pathol. 2016; 145(6):826-836.

30. Koganemaru S, Inoshita N, Miura Y, et al. Prognostic value of programmed death-ligand 1 expression in patients with stage III colorectal cancer. Cancer Sci. 2017;108(5):853-858.

31. Schalper KA, Brown J, Carvajal-Hausdorf D, et al. Objective measurement and clinical significance of TILs in non-small cell lung cancer. J Natl Cancer Inst. 2015;107(3). pii: dju435.

32. Horne ZD, Jack R, Gray ZT, et al. Increased levels of tumor-infiltrating lymphocytes are associated with improved recurrence-free survival in stage 1A non-small-cell lung cancer. J Surg Res. 2011;171(1):1-5.
OncoTargets and Therapy

\section{Publish your work in this journal}

OncoTargets and Therapy is an international, peer-reviewed, open access journal focusing on the pathological basis of all cancers, potential targets for therapy and treatment protocols employed to improve the management of cancer patients. The journal also focuses on the impact of management programs and new therapeutic agents and protocols on

\section{Dovepress}

patient perspectives such as quality of life, adherence and satisfaction The manuscript management system is completely online and includes a very quick and fair peer-review system, which is all easy to use. Visit http://www.dovepress.com/testimonials.php to read real quotes from published authors. 